\title{
Alterations of Fatty Acid Profile May Contribute to Dyslipidemia in Chronic Kidney Disease by Influencing Hepatocyte Metabolism
}

\author{
Aleksandra Czumaj ${ }^{1}{ }^{\circledR}$, Tomasz Śledziński ${ }^{1, *}$, Juan-Jesus Carrero ${ }^{2}$, Piotr Stepnowski ${ }^{3}$, \\ Malgorzata Sikorska-Wisniewska ${ }^{4}$, Michal Chmielewski ${ }^{4}$ and Adriana Mika ${ }^{1,3}$ \\ 1 Department of Pharmaceutical Biochemistry, Faculty of Pharmacy, Medical University of Gdansk, \\ 80-211 Gdansk, Poland; aczumaj@gumed.edu.pl (A.C.); adriana.mika@ug.edu.pl (A.M.) \\ 2 Medical Epidemiology and Biostatistics (MEB), Karolinska Institutet, SE-171 77 Stockholm, Sweden; \\ Juan.Jesus.Carrero@ki.se \\ 3 Department of Environmental Analysis, Faculty of Chemistry, University of Gdansk, 80-308 Gdansk, Poland; \\ piotr.stepnowski@ug.edu.pl \\ 4 Department of Nephrology, Transplantology and Internal Medicine, Faculty of Medicine, Medical University \\ of Gdansk, 80-214 Gdansk, Poland; gosia.sikorska@gmail.com (M.S.-W.); chmiel@gumed.edu.pl (M.C.) \\ * Correspondence: tomasz.sledzinski@gumed.edu.pl or tsledz@gumed.edu.pl; \\ Tel.: +48-583491479; Fax: +48-583491465
}

Received: 11 April 2019; Accepted: 17 May 2019; Published: 18 May 2019

check for updates

\begin{abstract}
Chronic kidney disease (CKD) is associated with atherogenic dyslipidemia. Our aim was firstly to investigate patterns of fatty acids (FA) composition through various stages of CKD, and secondly, to evaluate the effect of CKD-specific FA disturbances on the expression of genes related to lipid metabolism at a cellular level. Serum FA composition was analyzed in 191 patients with consecutive severity stages of CKD, and 30 healthy controls free from CKD. Next, HepG2 human hepatic cells were treated with major representatives of various FA groups, as well as with FA extracted from a mix of serums of controls and of CKD stage 5 patients. Across worsening stages of CKD severity, there was an increasing monounsaturated FA (MUFA) content. It was associated with a concomitant decrease in n-3 and n-6 polyunsaturated FA. The incubation of hepatocytes with FA from CKD patients (compared to that of healthy subjects), resulted in significantly higher mRNA levels of genes involved in FA synthesis (fatty acid synthase (FASN) increased $13.7 \pm 3.5$ times, stearoyl-CoA desaturase 1 (SCD1) increased $4.26 \pm 0.36$ times), and very low density lipoprotein (VLDL) formation (apolipoprotein B (ApoB) increased $7.35 \pm 1.5$ times, microsomal triacylglycerol transfer protein $(M T T P)$ increased $2.74 \pm 0.43$ times). In conclusion, there were progressive alterations in serum FA composition of patients with CKD. These alterations may partly contribute to CKD hypertriglyceridemia by influencing hepatocyte expression of genes of lipid synthesis and release.
\end{abstract}

Keywords: chronic kidney disease; fatty acids; lipids; lipogenesis; hypertriglyceridemia; hepatocyte

\section{Introduction}

Chronic kidney disease (CKD) is accompanied by lipid disorders due to metabolic alterations associated with the retention of uremic solutes, as well as to dietary restrictions and impaired food intake resulting from chronic intoxication [1]. Dialysis does not normalize the lipid profile, and in patients after renal transplantation it may even further aggravate, mainly because of the immunosuppressive treatment. CKD-dyslipidemia likely contributes to increased cardiovascular risk [1].

Lipids count over 1.6 million species and most of them contain fatty acids (FA) in their structure [2]. Serum FA concentration and FA composition has a significant effect in maintaining the metabolic 
balance in the organism. Disturbed blood FA profile is associated with oxidative stress, lipotoxicity and hypertriglyceridemia. Patients suffering from CKD are potentially prone to FA disorders due to metabolic alterations associated with the retention of uremic solutes, as well as to dietary restrictions and impaired food intake resulting from chronic intoxication.

Various studies, including ours, have reported alterations in the proportion of particular serum FA groups across more severe CKD stages, particularly a progressively lower n-3 polyunsaturated fatty acids (PUFA) content and higher monounsaturated fatty acids (MUFA) content [3-6]. The consequences of these changes are not known. Studies outside nephrology suggest that FA can modulate a number of cellular pathways involved in lipid and lipoprotein metabolism. In fact, they may regulate gene expression, and signal transduction cascades, e.g., through transcription factors including sterol regulatory binding proteins (SREBPs), peroxisome proliferator-activated receptors (PPARs), hepatocyte nuclear factor $4 \alpha(\mathrm{HNF} 4 \alpha)$, liver- $x$ receptors (LXR) and nuclear factor $\kappa B$ (NFkB) [7-11]. They also play important roles in numerous physiological processes, including energy metabolism (secretion of insulin, glucagon and incretins) and inflammatory responses (cytokine production) [12].

While it is well-established that dietary FA can impact on the regulation of genes involved in lipid metabolism $[13,14]$, it is conceivable that CKD-induced changes in serum FA composition may also play a role at this level. The aim of this study was firstly to investigate patterns of FA composition through various stages of CKD. Secondly, given that liver is the major organ responsible for lipid metabolism, we studied the effect that these CKD-specific FA disturbances might have on the expression of genes related to lipid metabolism in hepatocytes.

\section{Results}

\subsection{Clinical Data}

Characteristics of the included patients with CKD, undergoing hemodialysis (HD) or peritoneal dialysis (PD), and after renal transplantation (Tx) are presented in Table 1. Across worsening CKD stages, we noted increased concentrations of triacylglycerols (TAG), glucose, insulin, homeostatic model of insulin resistance (HOMA IR), C-reactive protein (CRP) and $\mathrm{K}^{+}$. In addition, hemoglobin, albumin and HDL cholesterol were decreased, while total and LDL cholesterol remained fairly stable (Table 1).

The serum FA profile showed significant alterations in the composition of many FA families across worsening stages of CKD (Table 2). The most prominent alterations were an increase in MUFA content and a decrease in n-3 and n-6 PUFA (Figure 1). Following a multivariable general linear model (GLM) analysis (adjusted by age, sex and diabetes mellitus), CKD stage emerged as a significant predictor of MUFA and PUFA proportions. Dialysis therapies did not seem to modify this pattern, except for the n-3 PUFA level in PD subjects (Figure 1). Obtained results also did not show considerable associations between the consumption of food products rich in MUFA, n-3 PUFA or n-6 PUFA and serum content of these FA in CKD patients (Table S1). Lipid lowering medications have not altered patients FA profile significantly. 
Table 1. Selected biochemical and anthropometric characteristics of the study subjects. ${ }^{*}$ statistically significant comparing to controls at $p<0.05$; \# $p<0.01$.

CKD—chronic kidney disease, HD - hemodialysis, PD—peritoneal dialysis, Tx — renal transplantation, eGFR—glomerular filtration rate, BUN—blood urea nitrogen,

TAG—triacyloglycerol, CRP—C-reactive protein, BMI—body mass index, HOMA IR—Homeostatic model assessment for insulin resistance, DM—diabetes mellitus,

CVD—cardiovascular disease, HP—hypertension.

\begin{tabular}{|c|c|c|c|c|c|c|c|c|}
\hline Item & Healthy Control & CKD 1-2 & CKD 3a & CKD $3 b$ & CKD 4-5 & HD & PD & Tx \\
\hline Age (years) & $55.57 \pm 1.34$ & $53.65 \pm 2.30$ & $60.57 \pm 2.32$ & $61.15 \pm 2.64$ & $61.00 \pm 2.81$ & $60.14 \pm 2.40$ & $51.27 \pm 2.18$ & $52.17 \pm 2.14$ \\
\hline Hemoglobin (g/dL) & $14.37 \pm 0.20$ & $14.23 \pm 0.21$ & $14.61 \pm 0.30$ & $13.24 \pm 0.39 *$ & $12.33 \pm 0.39^{\#}$ & $10.71 \pm 0.26^{\#}$ & $10.99 \pm 0.29^{\#}$ & $13.17 \pm 0.41^{\#}$ \\
\hline Creatinine (mg/dL) & $0.85 \pm 0.03$ & $0.83 \pm 0.03$ & $1.33 \pm 0.04^{\#}$ & $1.72 \pm 0.08^{\#}$ & $3.98 \pm 0.96^{\#}$ & $7.61 \pm 0.46^{\#}$ & $9.55 \pm 0.67^{\#}$ & $1.57 \pm 0.17^{\#}$ \\
\hline eGFR $\left(\mathrm{ml} / \mathrm{min} / 1.73 \mathrm{~m}^{2}\right)$ & $89.07 \pm 2.18$ & $88.05 \pm 2.49$ & $50.96 \pm 1.17^{\#}$ & $36.59 \pm 1.06^{\#}$ & $20.63 \pm 1.35^{\#}$ & $7.11 \pm 0.54^{\#}$ & $6.30 \pm 0.71^{\#}$ & $39.27 \pm 2.65^{\#}$ \\
\hline $\mathrm{BUN}(\mathrm{mmol} / \mathrm{L})$ & $15.92 \pm 0.67$ & $16.49 \pm 0.64$ & $21.63 \pm 0.88^{\#}$ & $31.69 \pm 1.94 \#$ & $48.34 \pm 3.49^{\#}$ & $53.81 \pm 3.00^{\#}$ & $57.68 \pm 3.54^{\#}$ & $31.32 \pm 3.29^{\#}$ \\
\hline TAG (mg/dL) & $129.80 \pm 10.83$ & $148.10 \pm 17.07$ & $151.48 \pm 14.48$ & $154.77 \pm 12.06$ & $161.32 \pm 15.32$ & $185.46 \pm 32.68$ & $187.41 \pm 14.60^{\#}$ & $215.33 \pm 37.46^{*}$ \\
\hline Total cholesterol $(\mathrm{mmol} / \mathrm{L})$ & $211.23 \pm 8.19$ & $212.85 \pm 7.32$ & $202.22 \pm 11.68$ & $199.62 \pm 10.33$ & $212.84 \pm 12.78$ & $184.25 \pm 10.04 *$ & $209.79 \pm 12.16$ & $217.46 \pm 11.62$ \\
\hline HDL cholesterol (mmol/L) & $54.30 \pm 2.58$ & $55.93 \pm 2.20$ & $48.43 \pm 2.66$ & $48.73 \pm 3.12$ & $48.26 \pm 3.53$ & $41.46 \pm 2.47^{\#}$ & $38.76 \pm 1.92 \#$ & $51.71 \pm 3.92$ \\
\hline LDL cholesterol (mmol/L) & $131.10 \pm 7.41$ & $126.29 \pm 6.28$ & $121.32 \pm 8.79$ & $119.96 \pm 9.30$ & $131.79 \pm 10.79$ & $111.67 \pm 9.87$ & $135.43 \pm 10.90$ & $125.80 \pm 10.87$ \\
\hline CRP (mg/L) & $2.80 \pm 0.90$ & $3.38 \pm 0.65$ & $3.38 \pm 0.77$ & $4.10 \pm 0.91$ & $4.59 \pm 1.43$ & $14.19 \pm 5.43 *$ & $9.05 \pm 3.45$ & $8.64 \pm 3.98$ \\
\hline Albumin $(\mathrm{g} / \mathrm{L})$ & $39.46 \pm 0.45$ & $38.70 \pm 1.06$ & $39.52 \pm 0.61$ & $37.78 \pm 0.76$ & $36.74 \pm 1.06^{*}$ & $31.96 \pm 0.68^{\#}$ & $31.60 \pm 0.84^{\#}$ & $37.92 \pm 0.86$ \\
\hline Glucose (mg/dL) & $102.55 \pm 4.44$ & $102.67 \pm 4.79$ & $114.00 \pm 5.35$ & $123.07 \pm 10.65$ & $125.16 \pm 11.34$ * & $112.96 \pm 12.13$ & $96.83 \pm 4.95$ & $116.17 \pm 10.10$ \\
\hline Insulin $(\mu \mathrm{U} / \mathrm{mL})$ & $11.77 \pm 0.96$ & $12.99 \pm 1.17$ & $15.80 \pm 3.39$ & $15.84 \pm 1.19^{*}$ & $21.05 \pm 6.99$ & $23.55 \pm 9.47$ & $15.13 \pm 4.20$ & $30.18 \pm 15.00$ \\
\hline $\mathrm{Na}^{+}(\mathrm{mmol} / \mathrm{L})$ & $140.27 \pm 0.42$ & $140.00 \pm 0.41$ & $140.10 \pm 0.34$ & $139.62 \pm 0.62$ & $140.68 \pm 0.78$ & $137.25 \pm 0.56^{\#}$ & $141.17 \pm 0.46$ & $139.54 \pm 0.68$ \\
\hline $\mathrm{K}^{+}(\mathrm{mmol} / \mathrm{L})$ & $4.36 \pm 0.05$ & $4.24 \pm 0.05$ & $4.50 \pm 0.09$ & $4.52 \pm 0.09$ & $4.81 \pm 0.09^{\#}$ & $5.09 \pm 0.15^{\#}$ & $4.42 \pm 0.10$ & $4.26 \pm 0.08$ \\
\hline BMI $\left(\mathrm{kg} / \mathrm{m}^{2}\right)$ & $27.52 \pm 0.67$ & $27.52 \pm 0.84$ & $28.63 \pm 1.05$ & $31.16 \pm 1.13 *$ & $27.54 \pm 1.18$ & $25.84 \pm 1.15$ & $26.16 \pm 0.77$ & $27.04 \pm 0.85$ \\
\hline HOMA IR & $1.93 \pm 0.42$ & $3.13 \pm 0.40^{*}$ & $3.73 \pm 1.29$ & $4.32 \pm 0.53^{\#}$ & $6.14 \pm 3.57$ & $5.49 \pm 2.08^{*}$ & $4.44 \pm 1.98$ & $7.15 \pm 2.65 *$ \\
\hline \multicolumn{9}{|l|}{ Concomitant diseases (\%) } \\
\hline Diabetes mellitus (DM) & $3.33 \pm 3.33$ & $12.50 \pm 5.30$ & $27.27 \pm 9.50 *$ & $37.04 \pm 9.47^{\#}$ & $42.11 \pm 11.64^{\#}$ & $35.71 \pm 9.22^{\#}$ & $16.67 \pm 6.92$ & $33.33 \pm 9.83^{\#}$ \\
\hline Cardiovascular disease (CVD) & $3.33 \pm 3.33$ & $15.00 \pm 5.72$ & $40.91 \pm 10.49^{\#}$ & $44.44 \pm 9.75^{\#}$ & $42.11 \pm 11.64^{\#}$ & $57.14 \pm 9.52^{\#}$ & $36.67 \pm 8.95^{\#}$ & $33.33 \pm 9.83^{\#}$ \\
\hline Hypertension (HP) & $33.33 \pm 8.75$ & $70.00 \pm 7.34^{\#}$ & $95.65 \pm 4.45^{\#}$ & $92.59 \pm 5.14 \#$ & $100.00 \pm 7.65^{\#}$ & $92.86 \pm 4.96^{\#}$ & $100 \pm 0.00^{\#}$ & $100.00 \pm 0.00^{\#}$ \\
\hline
\end{tabular}


Table 2. The percent content of the main classes of fatty acids in serum of patients at successive stages of chronic kidney disease (CKD). The data are presented as fatty acid proportions (\%). ${ }^{*}$ statistically significant comparing to controls at $p<0.05$; $\#<<0.01$. SFA—saturated fatty acids, MUFA—monounsaturated fatty acids, PUFA-polyunsaturated fatty acids.

\begin{tabular}{|c|c|c|c|c|c|c|c|c|}
\hline Item & Healthy Control & CKD 1-2 & CKD 3a & CKD 3b & CKD 4-5 & HD & PD & $T x$ \\
\hline 14:0 & $1.18 \pm 0.06$ & $1.18 \pm 0.06$ & $1.26 \pm 0.07$ & $1.18 \pm 0.08$ & $1.06 \pm 0.06$ & $1.17 \pm 0.08$ & $0.98 \pm 0.06^{*}$ & $1.33 \pm 0.12$ \\
\hline $16: 0$ & $23.00 \pm 0.33$ & $23.04 \pm 0.30$ & $23.64 \pm 0.50$ & $23.21 \pm 0.30$ & $23.50 \pm 0.39$ & $23.51 \pm 0.39$ & $22.79 \pm 0.37$ & $23.86 \pm 0.45$ \\
\hline 18:0 & $7.23 \pm 0.12$ & $7.15 \pm 0.09$ & $7.12 \pm 0.16$ & $7.02 \pm 0.16$ & $6.79 \pm 0.18 *$ & $7.04 \pm 0.20$ & $6.87 \pm 0.18$ & $6.72 \pm 0.13^{\#}$ \\
\hline Other SFA & $1.57 \pm 0.04$ & $2.08 \pm 0.09 \#$ & $1.91 \pm 0.11^{\#}$ & $1.98 \pm 0.11^{\#}$ & $2.00 \pm 0.11^{\#}$ & $2.04 \pm 0.10^{\#}$ & $1.48 \pm 0.07$ & $2.27 \pm 0.10^{\#}$ \\
\hline Total SFA & $32.97 \pm 0.34$ & $33.44 \pm 0.36$ & $33.93 \pm 0.14$ & $33.38 \pm 0.44$ & $33.36 \pm 0.49$ & $33.75 \pm 0.38$ & $32.12 \pm 0.51$ & $34.18 \pm 0.57$ \\
\hline 14:1 & $0.07 \pm 0.01$ & $0.07 \pm 0.01$ & $0.08 \pm 0.01$ & $0.07 \pm 0.01$ & $0.06 \pm 0.01$ & $0.07 \pm 0.01$ & $0.05 \pm 0.00^{\#}$ & $0.08 \pm 0.01$ \\
\hline $16: 1$ & $1.93 \pm 0.30$ & $2.80 \pm 0.17^{\#}$ & $2.41 \pm 0.32$ & $2.85 \pm 0.17^{\#}$ & $2.22 \pm 0.35$ & $2.78 \pm 0.22 *$ & $2.76 \pm 0.15^{*}$ & $3.06 \pm 0.17^{\#}$ \\
\hline $18: 1$ & $26.04 \pm 0.55$ & $26.04 \pm 0.47$ & $25.68 \pm 1.29$ & $27.56 \pm 0.59$ & $28.57 \pm 0.49^{\#}$ & $29.24 \pm 0.75$ \# & $29.81 \pm 0.58^{\#}$ & $28.00 \pm 0.64$ * \\
\hline Other MUFA & $0.47 \pm 0.02$ & $0.46 \pm 0.01$ & $0.47 \pm 0.02$ & $0.51 \pm 0.02$ & $0.57 \pm 0.07$ & $0.53 \pm 0.02$ & $0.47 \pm 0.02$ & $0.53 \pm 0.03$ \\
\hline Total MUFA & $29.60 \pm 0.64$ & $29.59 \pm 0.54$ & $30.39 \pm 0.65$ & $31.09 \pm 0.62$ & $32.19 \pm 0.54^{\#}$ & $32.81 \pm 0.87^{\#}$ & $33.10 \pm 0.68^{\#}$ & $31.67 \pm 0.73$ * \\
\hline $18: 3 n-3$ & $0.35 \pm 0.02$ & $0.30 \pm 0.02$ & $0.29 \pm 0.03$ & $0.27 \pm 0.02 \#$ & $0.24 \pm 0.03^{\#}$ & $0.20 \pm 0.02 \#$ & $0.24 \pm 0.02 \#$ & $0.21 \pm 0.02^{\#}$ \\
\hline $20: 5 n-3$ & $1.11 \pm 0.13$ & $0.85 \pm 0.07$ & $0.81 \pm 0.07$ & $0.77 \pm 0.07$ * & $0.77 \pm 0.11$ & $0.72 \pm 0.07$ * & $0.95 \pm 0.05$ & $0.60 \pm 0.06^{\#}$ \\
\hline $22: 6 n-3$ & $1.14 \pm 0.08$ & $1.01 \pm 0.06$ & $1.03 \pm 0.08$ & $1.06 \pm 0.06$ & $0.96 \pm 0.10$ & $0.92 \pm 0.09$ & $1.38 \pm 0.08^{*}$ & $0.88 \pm 0.08 *$ \\
\hline Other n-3 PUFA & $0.39 \pm 0.00$ & $0.36 \pm 0.01$ & $0.34 \pm 0.02 *$ & $0.37 \pm 0.02$ & $0.34 \pm 0.01^{\#}$ & $0.36 \pm 0.01$ & $0.50 \pm 0.02 \#$ & $0.32 \pm 0.02{ }^{\#}$ \\
\hline Total n-3 PUFA & $2.99 \pm 0.21$ & $2.52 \pm 0.13$ & $2.47 \pm 0.15$ & $2.48 \pm 0.13$ & $2.31 \pm 0.20$ * & $2.19 \pm 0.16^{\#}$ & $3.08 \pm 0.13$ & $2.01 \pm 0.13^{\#}$ \\
\hline $18: 2 n-6$ & $24.90 \pm 0.64$ & $25.14 \pm 0.60$ & $24.31 \pm 0.99$ & $23.85 \pm 0.76$ & $23.99 \pm 0.95$ & $22.25 \pm 0.64$ \# & $22.73 \pm 0.67 *$ & $24.13 \pm 0.82$ \\
\hline $20: 4 n-6$ & $5.56 \pm 0.20$ & $5.05 \pm 0.17$ & $4.93 \pm 0.22 *$ & $5.11 \pm 0.17$ & $4.66 \pm 0.27^{\#}$ & $4.93 \pm 0.25$ & $5.15 \pm 0.20$ & $4.03 \pm 0.17^{\#}$ \\
\hline Other n-6 PUFA & $1.44 \pm 0.04$ & $1.37 \pm 0.04$ & $1.30 \pm 0.04$ * & $1.23 \pm 0.05^{\#}$ & $1.10 \pm 0.07^{\#}$ & $1.13 \pm 0.06^{\#}$ & $1.43 \pm 0.04$ & $1.12 \pm 0.04^{\#}$ \\
\hline Total n-6 PUFA & $31.90 \pm 0.67$ & $31.57 \pm 0.67$ & $30.54 \pm 0.24$ & $30.19 \pm 0.77$ & $29.72 \pm 0.90$ & $28.31 \pm 0.72 \#$ & $29.31 \pm 0.77^{*}$ & $29.28 \pm 0.91$ * \\
\hline
\end{tabular}




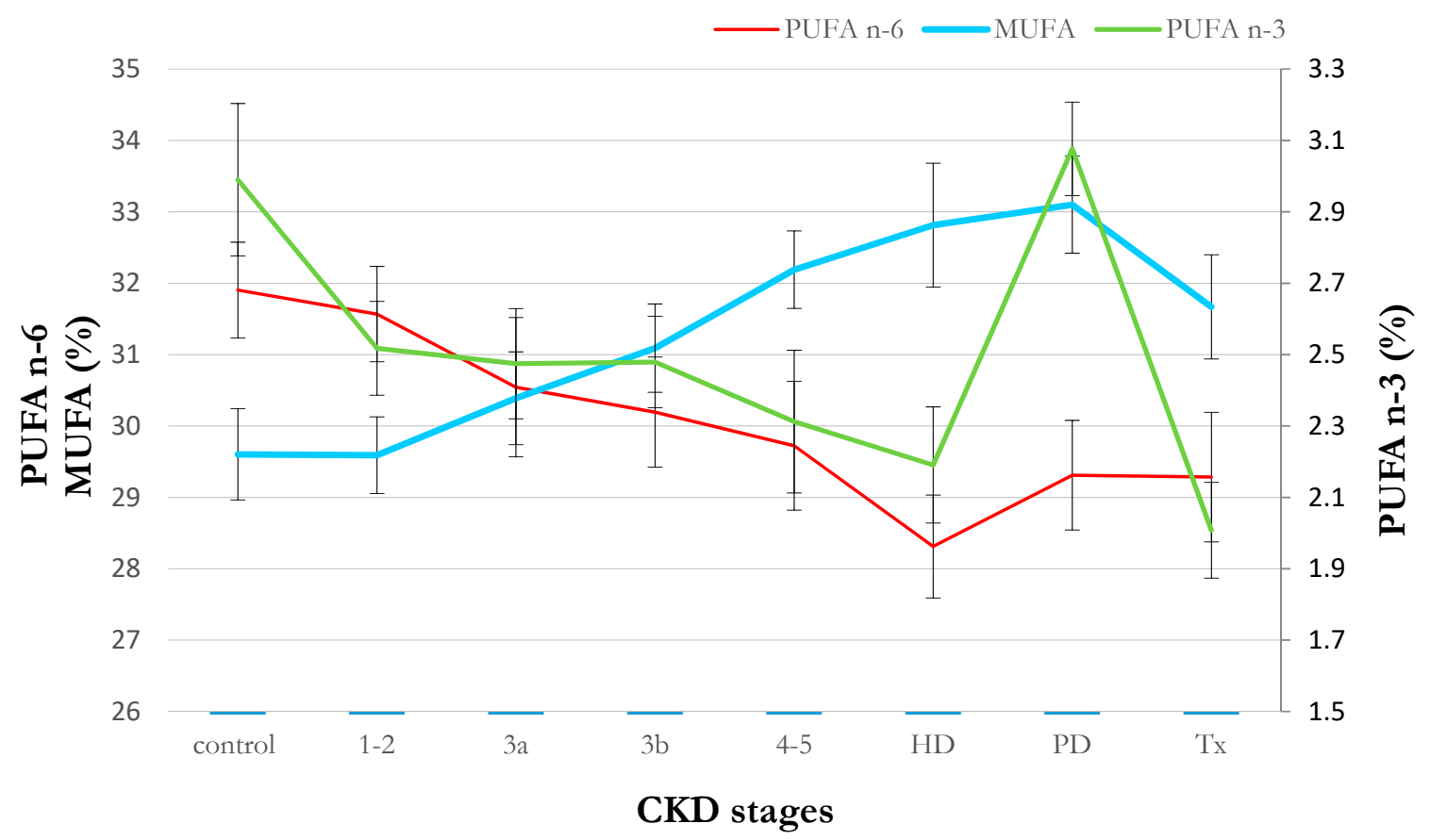

Figure 1. Serum proportion of n-3, n-6 polyunsaturated fatty acids (PUFA) and monounsaturated fatty acids (MUFA) in chronic kidney disease (CKD) patients with consecutive stages of disease. Data are shown as mean \pm SEM. ${ }^{*} p<0.01$; ${ }^{\#} p<0.05$ comparing to healthy control group. CKD—chronic kidney disease; HD—hemodialysis; PD—peritoneal dialysis; Tx — renal transplantation; PUFA—-polyunsaturated fatty acids; MUFA—-monounsaturated fatty acids.

\subsection{Cell Studies In Vitro}

To examine if the observed alterations in the serum FA composition in CKD patients had an impact on dyslipidemia related genes, we treated human HepG2 hepatocytes with the main MUFA, n-3 and n-6 PUFA representatives—oleic acid (OA), docosahexaenoic acid (DHA) and arachidonic acid (AA), respectively.

Incubation with OA resulted in a significant increase in mRNA levels of genes encoding enzymes of fatty acid synthesis and desaturation (acetyl-CoA carboxylase (ACC), fatty acid synthase (FASN) and stearoyl-CoA desaturase (SCD1)), TAG synthesis (diacylglycerol O-transferase (DGAT1)), as well as microsomal TAG transfer protein (MTTP) involved in very low density lipoprotein (VLDL) formation (Figure 2A). There was also a trend towards an increased mRNA level of SREBP1-transcription factor promoting lipid synthesis, and apolipoproteins A1 (ApoA1) and B $(A p o B)$. In contrast, mRNA levels of FA elongase 6 (ELOVL6) decreased (Figure 2A). All these changes appeared to be dose dependent.

Incubation with AA led to a decrease in SCD1, ELOVL6, DGAT, MTTP and SREBP1, it did not change the expression of $A C C$, and apolipoproteins, and increased FASN mRNA levels (Figure 2B). Incubation of hepatocytes with DHA resulted in generally the same changes of genes expression as the treatment with AA (Figure 2C). 

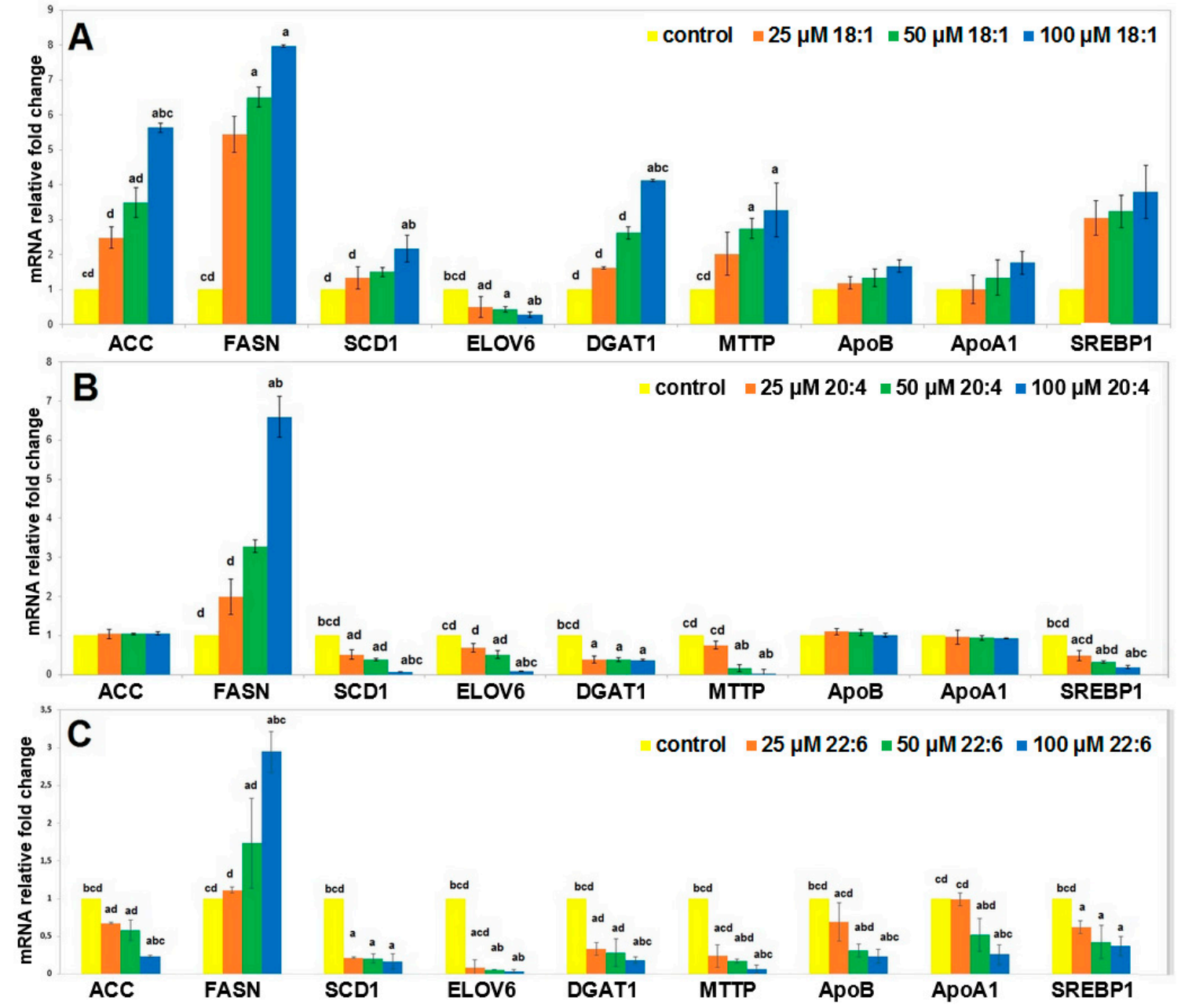

Figure 2. mRNA levels for selected genes expressed in HepG2 cells supplemented with various concentrations of oleic acid (OA; A), arachidonic acid (AA; B) and docosahexaenoic acid (DHA; C). Data are shown as mean \pm SEM. Groups significantly different by one-way ANOVA $(p<0.05)$ : $\mathrm{a}-p<0.05$ compared to the control; $\mathrm{b}-p<0.05$ compared to the cells treated by $25 \mu \mathrm{M}$ FA; $c-p<0.05$ compared to the cells treated by $50 \mu \mathrm{M}$ FA and $\mathrm{d}-p<0.05$ compared to the cells treated by $100 \mu \mathrm{M}$ FA. Data are presented as means \pm SEM. ACC - acetyl-coenzyme A carboxylase, FASN-fatty acid synthase, SCD1—stearoyl-CoA desaturase, ELOVL6-fatty acid elongase 6, DGAT1-diacylglycerol O-acyltransferase 1, MTTP-microsomal triglyceride transfer protein, ApoB-apolipoprotein B, ApoA1-apolipoprotein A1, SREBP1—sterol regulatory element-binding protein 1 . - control; $-25 \mu \mathrm{M}$ $\mathrm{FA} ;-50 \mu \mathrm{M}$ FA; $-100 \mu \mathrm{M}$ FA.

Summarizing this first experiment, OA generally increased the expression of genes related to liver lipid synthesis and release (except ELOVL6), whereas AA and DHA decreased or did not influence the expression of these genes (except FASN).

\subsection{Cell Studies Ex Vivo}

As a next step, we extracted FA from pooled serum of 12 CKD stage 5 patients and of 12 healthy controls, and we incubated hepatocytes with such FA preparation. The composition of the CKD- and control-FA preparations was evaluated by GC-MS and presented in Table 3. FA preparations extracted from CKD patients had higher MUFA and lower n-3 as well as n-6 PUFA content in comparison to those of control individuals. 
Table 3. Content of fatty acids in extracts from patients with chronic kidney disease (CKD) stage 5 and healthy controls serum mixes used for hepatocyte treatment in vitro. The data are presented as a fatty acid proportion (\%). SFA—saturated fatty acids, MUFA-monounsaturated fatty acids, PUFA-polyunsaturated fatty acids.

\begin{tabular}{ccc}
\hline Item & Control & CKD Stage 5 \\
\hline $14: 0$ & 1.02 & 1.33 \\
$16: 0$ & 22.72 & 26.84 \\
$18: 0$ & 7.99 & 6.46 \\
Other SFA & 1.56 & 1.10 \\
Total SFA & 32.27 & 35.73 \\
$14: 1$ & 0.04 & 0.09 \\
$16: 1$ & 1.74 & 3.29 \\
$18: 1$ & 23.59 & 29.59 \\
Other MUFA & 0.80 & 0.62 \\
Total MUFA & 26.17 & 33.85 \\
$18: 3 n-3$ & 0.23 & 0.20 \\
20:5n-3 & 0.94 & 0.66 \\
22:6n-3 & 1.17 & 0.93 \\
Other n-3 PUFA & 0.37 & 0.30 \\
Total n-3 PUFA & 2.71 & 2.09 \\
18:2n-6 & 30.03 & 22.76 \\
20:4n-6 & 6.03 & 4.28 \\
Other n-6 PUFA & 1.5 & 0.93 \\
Total n-6 PUFA & 37.56 & 27.97 \\
\hline
\end{tabular}

Compared to the FA mixture of healthy subjects, incubation of HepG2 with FA from CKD patients resulted in significantly increased mRNA levels of genes involved in fatty acid synthesis, desaturation, elongation and VLDL formation, as well as the SREBP1 gene (Figure 3). On the other hand, ApoA1 mRNA level was lower after incubating with CKD FA mixture. Finally, the concentration of TAG in the culture medium after $48 \mathrm{~h}$ culture of cells treated with the CKD FA mixture was approximately six-fold higher than following incubation with FA mixture from controls (Figure 4).

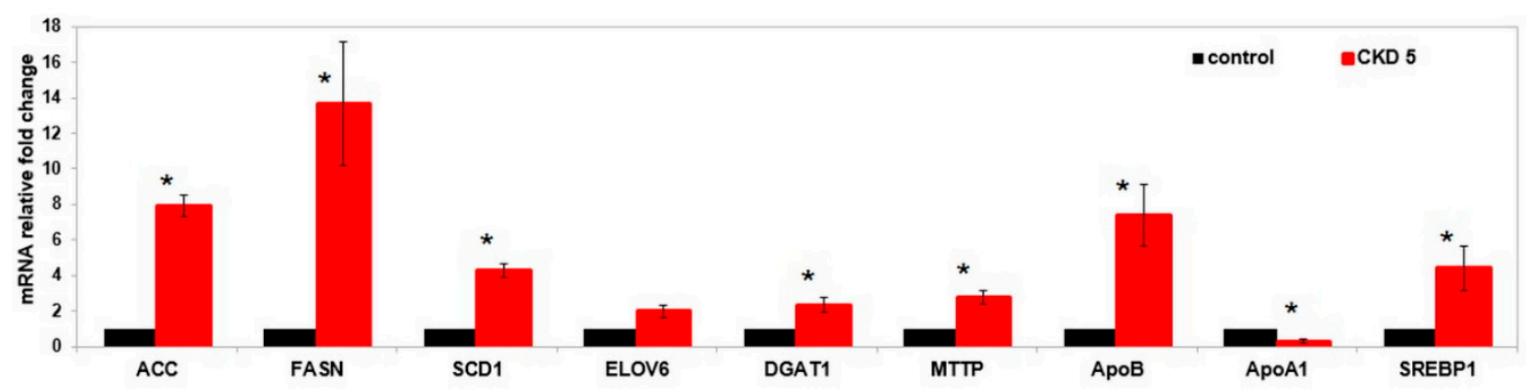

Figure 3. mRNA levels for selected genes expressed in HepG2 cells supplemented with fatty acids (FA) from control patients (control) and CKD stage 5 patients (CKD 5) serum. ${ }^{*} p<0.05$ compared to the control. Data are presented as means \pm SEM. ACC - acetyl-coenzyme A carboxylase, FASN-fatty acid synthase, SCD1—stearoyl-CoA desaturase, ELOVL6 - fatty acid elongase 6, DGAT1—diacylglycerol O-acyltransferase 1, MTTP-microsomal triglyceride transfer protein, $A p o B$-apolipoprotein B, ApoA1—apolipoprotein A1, SREBP1—sterol regulatory element-binding protein 1. 




Figure 4. Concentration of TAG in culture medium after $48 \mathrm{~h}$ incubation with FA mixtures. Data are shown as mean $\pm \mathrm{SEM},{ }^{*} p<0.05$.

\section{Discussion}

Although previous studies have evaluated disorders in FA profile in patients with CKD, they have been usually limited to dialysis patients only [3-5,15]. In contrast, our study provides a comprehensive overview of changes in serum FA composition across the natural course of CKD progression. We show that across worsening stages of CKD severity, there was an increasing MUFA and gradually decreasing n-3 and n-6 PUFA content. Our recent study revealed that increased serum MUFA in CKD patients is associated with various risk factors of cardiovascular disease, primarily with hypertriglyceridemia [6]. Kidney transplantation did not result in full normalization of these derangements.

The novelty of our study is the evaluation of the impact of these CKD-specific FA alteration on lipid metabolism. We used an in vitro model of hepatocytes, given that the liver is the main organ involved in lipid metabolism, observing that oleic acid, the major MUFA representative, resulted in an increased expression of lipogenic genes (ACC, FASN, SCD1, DGAT1), as well as genes related to VLDL production and release $(A p o B, M T T P)$. Such changes have been reported to result from an increased expression of SREBP1-an upstream regulator of lipogenic genes [16]. It should be noted that increased expression of SREBP1 has been found in adipose tissue of rats with experimental renal failure in our previous studies [17]. Our results are consistent with these obtained recently by Patel et al. [18] who also found increased mRNA levels of SPREBP1, ACC and FASN in hepatocytes treated with OA, although these authors used much higher OA concentrations in culture $(1 \mathrm{mM})$, in comparison to our study $(25-100 \mu \mathrm{M})$.

In contrast, hepatocyte treatment with arachidonic acid (major n-6 PUFA representative) as well as docosahexaenoic acid (major n-3 PUFA representative), decreased or did not materially change mRNA levels of most of these genes. Only FASN mRNA level increased significantly after treatment with $100 \mu \mathrm{M}$ AA, a finding that seemed counterintuitive, given the behavior of the rest of the genes in the same pathway. Decreased hepatic de novo lipogenesis following n-6 PUFA treatment was also described by Caputo et al. [19] and Mater et al. [20].

Altogether, this evidence suggests that CKD-specific FA alterations might (by influencing liver metabolism) increase lipid production and release, leading to CKD-associated hypertriglyceridemia. However, an important limitation in performing this kind of experiments is the use of a single FA for cell incubation rather than the whole FA spectrum that contains more than 30 different FA [21]. Since each FA can influence liver cells, the CKD-specific alterations induced by the FA profile should ideally be considered globally. To tackle this, we used a pool preparation of FA isolated from serum of healthy subjects and patients with CKD stage 5. This experiment revealed that the pathological serum FA profile of CKD patients, including increased MUFA and decreased PUFA, might induce lipid synthesis and release in liver. Finally, increased triacylglycerol concentrations in culture media from cells treated with FA from CKD patients confirmed the functional end-point of changes in lipogenic 
genes expression. A schematic representation of our observations and working hypothesis is shown in Figure 5.

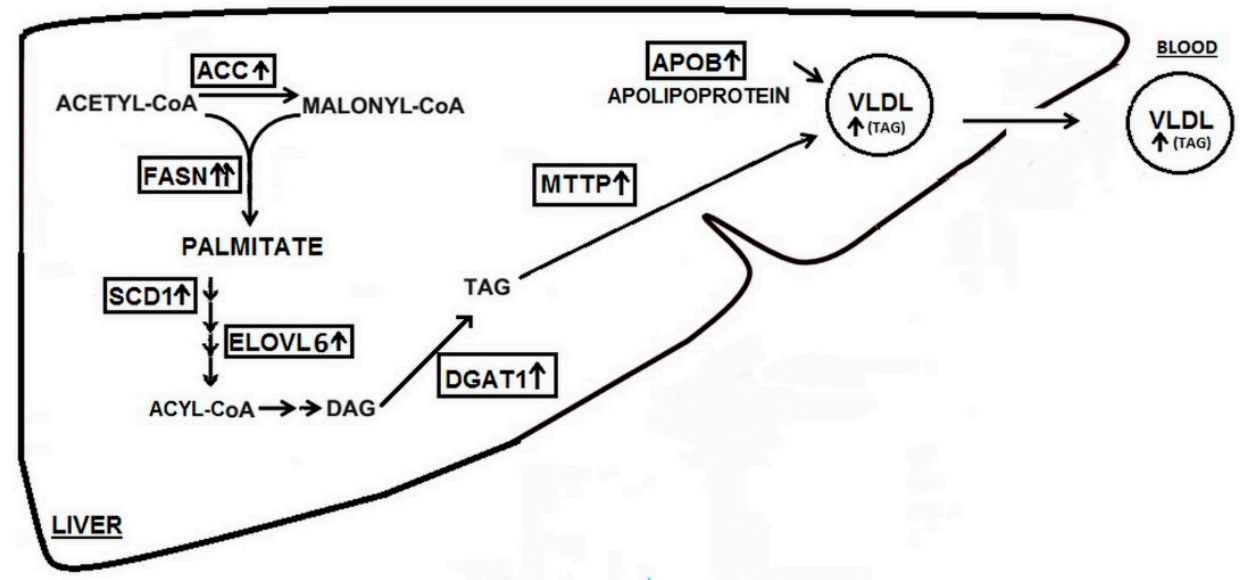

Figure 5. Scheme illustrating the plausible effects of fatty acids extracted from chronic kidney disease (CKD) patients' serum on the expression of genes associated with lipid synthesis and release in hepatocyte. ACC-acetyl-coenzyme A carboxylase, FASNfatty acid synthase, SCD1—stearoyl-CoA desaturase, ELOVL6-fatty acid elongase 6, DGAT1—diacylglycerol $O$-acyltransferase 1, MTTP-microsomal triglyceride transfer protein, ApoB-apolipoprotein B, DAG—diacylglycerol, TAG—triacylglycerol, VLDL—very low density lipoprotein.

Considering that MUFA are the main FA in triacylglycerols included in VLDL [22,23], we speculate that a self-perpetuating mechanism arises, in which the more MUFA in the patient's serum, the greater the production of triacylglycerols in the liver. This effect might contribute to hypertriglyceridemia, constantly observed in CKD patients. Increased triglyceride concentration is an acknowledged risk factor for cardio-vascular disease in the general population. Therefore, a gradual increase in MUFA across the CKD stages, combined with low PUFA content, demonstrated in the present study, might result in an augmented risk of cardio-vascular complications. Dietary modifications could diminish this risk, although this remains to be determined.

Treatment of hepatocytes with FA from CKD patients resulted also in an increased SREBP1 mRNA level, confirming its role in the induction of liver lipogenesis in CKD. Other authors reported that SREBP1 activity in the liver can be modulated by various FA [24]. The role of SREBPs in CKD dyslipidemia was also reported earlier by our group, on the basis of an experimental renal failure in animal models [16]. The upregulation of lipogenic enzymes was also reported in a rat renal failure model by Jin et. al. [25].

The study has limitations that ought to be underlined. First, we include a cross-sectional evaluation, therefore results present FA disorders across various CKD stages with no inference on temporality within disease progression or causation. A study design in which the same patients are followed and repeatedly sampled throughout their disease progression would have been preferred, but taken potentially decades to complete. Moreover, the large heterogeneity of the study groups with multiple CKD etiologies and treatments presents a challenge to a comparative study. However, strong associations between consecutive CKD stages and gradually increasing disturbances of the FA profile suggest that it is CKD itself that is responsible for the observed FA changes. Moreover, our observational analysis is coupled with well-designed cell culture experiments that support our hypothesis.

Finally, the studied cohort is relatively small, but even with these patient numbers the presented associations among evaluated variables are strong and convincing.

In conclusion, our study shows that the progressive alterations of serum FA composition in the course of CKD might contribute to CKD related hypertriglyceridemia through influencing the expression of genes of lipid synthesis and release in the hepatocyte. 


\section{Materials and Methods}

\subsection{Patients}

The first part of this study included a cohort of patients with CKD recruited from the Outpatient Unit of the Department of Nephrology, Transplantology and Internal Medicine in the Medical University of Gdansk. The cohort involved 191 patients, aged 18-70, with CKD stages 1-2 $(n=40), 3 \mathrm{a}(n=23)$, $3 \mathrm{~b}(n=27), 4-5(n=19)$, undergoing hemodialysis $(n=28)$, peritoneal dialysis $(n=30)$ and renal transplantation $(n=24)$. The etiology of CKD was diabetes (24 patients), glomerulonephritis (32), hypertension (25), anemia (6), autosomal dominant polycystic kidney disease (13), vasculitis (5), systemic lupus erythematosus (5), obstructive (18) or other (63). Ninety one CKD patients used statins, and four used fibrates. In addition, we recruited a group of control subjects of similar age and sex and free from any kidney damage $(n=30)$. Subjects using FA supplements were excluded from the study. The study was performed in agreement with the principles of the Declaration of Helsinki of the World Medical Association. Experimental protocols received approval from the Local Bioethics Committee at the Medical University of Gdansk (protocol no. NKEBN/614/2013-2014 issued on 28 May 2014) and informed consents were obtained from patients and healthy volunteers. All laboratory tests were carried out at the Central Clinical Laboratory of the Medical University of Gdansk.

The CKD stage assessment was based on the estimated glomerular filtration rate (eGFR) calculated with the CKD-EPI formula [26]. Presence of cardiovascular disease (CVD), diabetes mellitus (DM) and hypertension was based on the medical records. Body mass index (BMI) was calculated as the body mass divided by the square of the body height, and homeostatic model assessment for insulin resistance (HOMA IR) as glucose concentration $(\mathrm{mg} / \mathrm{dL})$ multiplied by insulin $(\mu \mathrm{U} / \mathrm{mL})$ and divided by 405. Blood was donated by the patients and the controls after an overnight fast. Dietary habits were investigated with the use of FFQ6 (food frequency questionnaire), the most common dietary assessment tool used in large epidemiologic studies of diet and health and validated for the Polish population [27]. FFQ is a tool for the evaluation of frequency (times/person/day) and amount (g/person/day) of food consumed during a year. The frequency of product consumption is determined by respondents by free pointing to habitual intake frequency from a list of 55 line items where each line item is defined by a series of foods or beverages.

\subsection{Lipid Extraction and FAME Analysis}

Total lipids in serum were extracted using the method described by Folch et al. [28]. Lipid samples were hydrolyzed with $0.5 \mathrm{M} \mathrm{KOH}$ in methanol and FA methyl esters (FAMEs) were prepared using $10 \%$ Boron Trifluoride reagent (BF3/methanol). After the evaporation of n-hexane, FAMEs were dissolved in dichloromethane and analyzed on a GC-MS QP-2010 SE (SHIMADZU Kioto, Japan) [29]. Compounds were separated on a $30 \mathrm{~m} \times 0.25 \mathrm{~mm}$ i.d., HP-5 capillary column (film thickness $0.25 \mu \mathrm{m}$ ). The temperature of the column was programmed from $60{ }^{\circ} \mathrm{C}$ to $300{ }^{\circ} \mathrm{C}$ at a rate of $4{ }^{\circ} \mathrm{C} \mathrm{min}{ }^{-1}$ with helium as carrier gas at a column head pressure of $60 \mathrm{kPa}$. 19-methyl-eicosanoate was used as the internal standard. All the chemicals and reagents were obtained from Sigma-Aldrich (Saint Louis, MO, USA). Exemplar chromatogram of GCMS fatty acid analysis from CKD patient is presented on supplementary Figure S1.

\subsection{Cell Cultures and Treatment}

The second part of the study involved in vitro and ex vivo experiments on the HepG2 human hepatoma cell line (ECACC, Salisbury, UK). Liver is the major organ responsible for lipid metabolism. Hep G2 cells are derived from liver tissue of human and are widely used as a model system for studies of liver metabolism. These cells secrete a variety of liver derived plasma proteins, and express the genes of lipid metabolism [30,31]. Cells were cultured in a humidified incubator at $37^{\circ} \mathrm{C}$ with $5 \% \mathrm{CO}_{2}$. HepG2 cells were maintained in minimum essential medium Eagle (MEM) containing 
$10 \%$ heat-inactivated fetal bovine serum (FBS), $2 \mathrm{mM}$ L-glutamine, $1 \%$ non-essential amino acids, 100 units/mL penicillin and $100 \mathrm{mg} / \mathrm{mL}$ streptomycin. The medium was replenished every two days.

Hepatocytes were treated with the following: OA, AA, DHA or FA extracted from a mix of serums obtained from control subjects and FA extracted from a mix of serums obtained from CKD patients with stage 5. OA, AA and DHA were purchased from Sigma-Aldrich (Saint Louis, MO, USA). FA preparations from patients and controls were prepared on site. From 12 randomly selected samples of healthy controls and 12 CKD stage 5 patients, $200 \mu \mathrm{L}$ of serum was taken and two solutions were prepared (control-mix and CKD-mix). Total lipids were extracted and hydrolyzed with $0.5 \mathrm{M} \mathrm{KOH}$ in methanol exactly as the samples for GC-MS analysis of FA but FA were not derivatized. Experimental stock solutions were prepared $48 \mathrm{~h}$ prior to experiments. All test components were conjugated with bovine serum albumin (BSA). Briefly, FA was dissolved in ethanol at $70{ }^{\circ} \mathrm{C}$, then mixed with $10 \%$ BSA at $55^{\circ} \mathrm{C}$ for $10 \mathrm{~min}$ to create conjugates. Cells were treated with FA conjugates dissolved in a serum-free medium. On the day after the passage, the cells were washed twice with $1 \mathrm{~mL}$ of phosphate-buffered saline (PBS), and then the specified experimental medium was added. Control cells were run in parallel in medium without test components. After $48 \mathrm{~h}$ incubation, the cells were washed twice with $1 \mathrm{~mL}$ of PBS and used for RNA isolation. OA, AA and DHA were tested in three variants of concentration of $25 \mathrm{uM}, 50 \mathrm{uM}$ and $100 \mathrm{uM}$. Concentration of FA mixes was adjusted to the concentrations observed in a patient's serum.

Total cellular RNA was isolated from HepG2 by GenElute Mammalian Total RNA Miniprep Kit (Sigma-Aldrich, (Saint Louis, MO, USA). The total RNA yields were determined for each sample by automated gel electrophoresis (Experion, Bio-Rad, Hercules, CA, USA). cDNA was synthesized from $1 \mu \mathrm{g}$ of total RNA using random hexamers and RevertAid reverse transcriptase according to the manufacturer's instructions. An aliquot of each cDNA synthesis reaction was subjected to PCR amplification using a CFX Connect Real-Time System (Bio-Rad, Hercules, CA, USA). The primers sequences are presented in supplemental Table S2. Results were normalized against a combination of $\beta$-actin and cyclophilin A.

\subsection{Triacylglycerols Content Analysis}

TAG content was determined in the HepG2 culture media using a triglyceride colorimetric assay kit (Cayman Chemical, Ann Arbor, MI, USA). Triacylglycerols in the samples were hydrolyzed by lipase to produce glycerol and free fatty acids. Released glycerol was subsequently measured by a coupled enzymatic reaction system (glycerol kinase, glycerol phosphate oxidase, peroxidase) that produce a brilliant purple quinoneimine dye. All samples and standards were measured in duplicate. The absorbance was measured at $540 \mathrm{~nm}$.

\subsection{Statistical Analyses}

Differences across the patient/control groups or cell experiments were analyzed, as appropriate, by a t-test or analysis of variance (ANOVA) followed by a post-hoc correction (Bonferroni) in the case of multi-group comparisons. To search for independent relationships between FA content and CKD, a general linear model (GLM) was applied. Statistical processing of the results was performed with the use of the statistical software STATISTICA PL v 13.0 (Statsoft, Kraków, Poland).

Supplementary Materials: Supplementary materials can be found at http://www.mdpi.com/1422-0067/20/10/ 2470/s1.

Author Contributions: Conceptualization A.C., A.M., T.S.; Investigation A.C., A.M.; Project administration T.S.; Resources M.S.-W.; Supervision M.C., P.S.; Visualization A.C., M.S.-W.; Writing一original draft A.C., A.M., T.S.; Writing-review \& editing J.-J.C., M.C., P.S., T.S.

Funding: This study was supported by a grant from the National Science Centre of Poland (grant no. NCN 2013/11/B/NZ5/00118, grant from the Ministry of Science and Higher Education of the Republic of Poland (DS 530-8615-D690-17), and grants from the Medical University of Gdansk (grants no. ST40, ST4), and by the project POWR.03.02.00-00-I026/17-00 co-financed with operational program Knowledge Education Development 2014-2020. 
Conflicts of Interest: The authors declare no conflict of interest. The funders had no role in the design of the study; in the collection, analyses, or interpretation of data; in the writing of the manuscript, or in the decision to publish the results.

\section{Abbreviations}

$\begin{array}{ll}\text { AA } & \text { Arachidonic acid } \\ \text { ACC } & \text { Acetyl-CoA carboxylase } \\ \text { ApoA1 } & \text { Apolipoprotein A1 } \\ \text { ApoB } & \text { Apolipoprotein B } \\ \text { BMI } & \text { Body mass index } \\ \text { BSA } & \text { Bovine serum albumin } \\ \text { BUN } & \text { Blood urea nitrogen } \\ \text { CDC } & \text { Chronic kidney disease } \\ \text { CRP } & \text { C-reactive protein } \\ \text { CVD } & \text { Cardiovascular disease } \\ \text { DAG } & \text { diacylglycerol } \\ \text { DHA } & \text { Docosahexaenoic acid } \\ \text { DGAT } & \text { Diacylglycerol O-transferase } \\ \text { DM } & \text { Diabetes mellitus } \\ \text { eGFR } & \text { Glomerular filtration rate } \\ \text { ELOVL } & \text { Elongase } \\ \text { FA } & \text { Fatty acids } \\ \text { FAME } & \text { Fatty acid methyl ester } \\ \text { FASN } & \text { Fatty acid synthase } \\ \text { FFQ } & \text { Food frequency questionnaire } \\ \text { GLM } & \text { General linear model } \\ \text { HD } & \text { Hemodialysis } \\ \text { HNF4 } \alpha & \text { Hepatocyte nuclear factor } 4 \alpha \\ \text { HP } & \text { Hypertension } \\ \text { HOMA IR } & \text { Homeostatic model assessment for insulin resistance } \\ \text { LXR } & \text { Liver-x receptors } \\ \text { MTTP } & \text { Microsomal TAG transfer protein } \\ \text { MUFA } & \text { Monounsaturated fatty acids } \\ \text { NFkB } & \text { Nuclear factor } \text { kB } \\ \text { OA } & \text { Oleic acid } \\ \text { PD } & \text { Peritoneal dialysis } \\ \text { PUFA } & \text { Polyunsaturated fatty acids } \\ \text { PPAR } & \text { Peroxisome proliferator-activated receptors } \\ \text { SCD } & \text { Stearoyl-CoA desaturase } \\ \text { SFA } & \text { Saturated fatty acids } \\ \text { SREBP } & \text { Sterol regulatory binding proteins } \\ \text { TAG } & \text { Triacylglycerol } \\ \text { Tx } & \text { Renal transplantation } \\ \text { VLDL } & \text { Very low density lipoprotein. } \\ \end{array}$

\section{References}

1. Chmielewski, M.; Carrero, J.J.; Lindholm, B. Lipid disorders in chronic kidney disease: Reverse epidemiology and therapeutic approach. Artic. J. Nephrol. 2008, 21, 635-644.

2. Li, L.; Han, J.; Wang, Z.; Liu, J.; Wei, J.; Xiong, S.; Zhao, Z. Mass Spectrometry Methodology in Lipid Analysis. Int. J. Mol. Sci. 2014, 15, 10492-10507. [CrossRef] [PubMed]

3. Sikorska-Wiśniewska, M.; Mika, A.; Śledziński, T.; Małgorzewicz, S.; Stepnowski, P.; Rutkowski, B.; Chmielewski, M. Disorders of serum omega-3 fatty acid composition in dialyzed patients, and their associations with fat mass. Ren. Fail. 2017, 39, 406-412. [CrossRef] [PubMed] 
4. Mika, A.; Stepnowski, P.; Chmielewski, M.; Malgorzewicz, S.; Kaska, L.; Proczko, M.; Ratnicki-Sklucki, K.; Sledzinski, M.; Sledzinski, T. Increased Serum Level of Cyclopropaneoctanoic Acid 2-Hexyl in Patients with Hypertriglyceridemia-Related Disorders; Increased Serum Level of Cyclopropaneoctanoic Acid 2-Hexyl in Patients with Hypertriglyceridemia-Related Disorders. Lipids 2016, 51, 867-873. [CrossRef] [PubMed]

5. Madsen, T.; Christensen, J.H.; Svensson, M.; Witt, P.M.; Toft, E.; Schmidt, E.B. Marine n-3 Polyunsaturated Fatty Acids in Patients With End-stage Renal Failure and in Subjects Without Kidney Disease: A Comparative Study. J. Ren. Nutr. 2011, 21, 169-175. [CrossRef] [PubMed]

6. Mika, A.; Sikorska-Wiśniewska, M.; Małgorzewicz, S.; Stepnowski, P.; Alicja, D.; Tomasz, Ś.; Chmielewski, M. Potential contribution of monounsaturated fatty acids to cardiovascular risk in chronic kidney disease. Pol. Arch. Med. wewnȩtrznej 2018, 128, 755-763. [CrossRef]

7. Desvergne, B.; Wahli, W. Peroxisome Proliferator-Activated Receptors: Nuclear Control of Metabolism*. Endocr. Rev. 1999, 20, 649-688. [PubMed]

8. Ou, J.; Tu, H.; Shan, B.; Luk, A.; Debose-Boyd, R.A.; Bashmakov, Y.; Goldstein, J.L.; Brown, M.S. Unsaturated fatty acids inhibit transcription of the sterol regulatory element-binding protein-1c (SREBP-1c) gene by antagonizing ligand-dependent activation of the LXR. PNAS 2001, 98, 6027-6032. [CrossRef]

9. Pawar, A.; Xu, J.; Jerks, E.; Mangelsdorf, D.J.; Jump, D.B. Fatty Acid Regulation of Liver X Receptors (LXR) and Peroxisome Proliferator-activated Receptor (PPAR) in HEK293 Cells*. J. Biol. Chem. 2002, 277, 39243-39250. [CrossRef] [PubMed]

10. Hertz, R.; Magenheim, J.; Berman, I.; Bar-Tana, J. Fatty acyl-CoA thioesters are ligands of hepatic nuclear factor-4 $\alpha$. Nature 1998, 392, 512-516. [CrossRef]

11. Wisely, G.B.; Miller, A.B.; Davis, R.G.; Thornquest, A.D.; Johnson, R.; Spitzer, T.; Sefler, A.; Shearer, B.; Moore, J.T.; Miller, A.B.; et al. Hepatocyte nuclear factor 4 is a transcription factor that constitutively binds fatty acids. Structure 2002, 10, 1225-1234. [CrossRef]

12. Hara, T.; Kashihara, D.; Ichimura, A.; Kimura, I.; Tsujimoto, G.; Hirasawa, A. Role of free fatty acid receptors in the regulation of energy metabolism. Biochim. Biophys. Acta - Mol. Cell Biol. Lipids 2014, 1841, 1292-1300. [CrossRef] [PubMed]

13. Clarke, S.D.; Armstrong, M.K.; Jump, D.B. Dietary Polyunsaturated Fats Uniquely Suppress Rat Liver Fatty Acid Synthase and S14 mRNA Content. J. Nutr. 1990, 120, 225-231. [CrossRef] [PubMed]

14. Landschulz, K.T.; Jump, D.B.; MacDougald, O.A.; Lane, M.D. Transcriptional control of the stearoyl-CoA desaturase-1 gene by polyunsaturated fatty acids. Biochem. Biophys. Res. Commun. 1994, 200, 763-768. [CrossRef] [PubMed]

15. Pazda, M.; Stepnowski, P.; Sledzinski, T.; Chmielewski, M.; Mika, A. Suitability of selected chromatographic columns for analysis of fatty acids in dialyzed patients. Biomed. Chromatogr. 2017, 31, e4006. [CrossRef]

16. Szolkiewicz, M.; Chmielewski, M.; Nogalska, A.; Stelmanska, E.; Swierczynski, J.; Rutkowski, B. The Potential Role of Sterol Regulatory Element Binding Protein Transcription Factors in Renal Injury. J. Ren. Nutr. 2007, 17, 62-65. [CrossRef] [PubMed]

17. Korczynska, J.; Stelmanska, E.; Nogalska, A.; Szolkiewicz, M.; Goyke, E.; Swierczynski, J.; Rutkowski, B. Upregulation of lipogenic enzymes genes expression in white adipose tissue of rats with chronic renal failure is associated with higher level of sterol regulatory element binding protein-1. Metabolism. 2004, 53, 1060-1065. [CrossRef]

18. Patel, T.P.; Rawal, K.; Soni, S.; Gupta, S. Swertiamarin ameliorates oleic acid induced lipid accumulation and oxidative stress by attenuating gluconeogenesis and lipogenesis in hepatic steatosis. Biomed. Pharmacother. 2016, 83, 785-791. [CrossRef]

19. Caputo, M.; De Rosa, M.C.; Rescigno, T.; Zirpoli, H.; Vassallo, A.; De Tommasi, N.; Torino, G.; Tecce, M.F. Binding of polyunsaturated fatty acids to LXR $\alpha$ and modulation of SREBP-1 interaction with a specific SCD1 promoter element. Cell Biochem. Funct. 2014, 32, 637-646. [CrossRef]

20. Mater, M.K.; Thelen, A.P.; Jump, D.B. Arachidonic acid and PGE2 regulation of hepatic lipogenic gene expression. J. Lipid Res. 1999, 40, 1045-1052.

21. Mika, A.; Stepnowski, P.; Kaska, L.; Proczko, M.; Wisniewski, P.; Sledzinski, M.; Sledzinski, T. A comprehensive study of serum odd- and branched-chain fatty acids in patients with excess weight. Obesity 2016, 24, 1669-1676. [CrossRef] [PubMed]

22. Liu, X.; Strable, M.S.; Ntambi, J.M. Stearoyl CoA Desaturase 1: Role in Cellular Inflammation and Stress. Adv. Nutr. 2011, 2, 15-22. [CrossRef] 
23. Ntambi, J.M.; Miyazaki, M. Regulation of stearoyl-CoA desaturases and role in metabolism. Prog. Lipid Res. 2004, 43, 91-104. [CrossRef]

24. Worgall, T.S.; Sturley, S.L.; Seo, T.; Osborne, T.F.; Deckelbaum, R.J. Polyunsaturated fatty acids decrease expression of promoters with sterol regulatory elements by decreasing levels of mature sterol regulatory element-binding protein. J. Biol. Chem. 1998, 273, 25537-25540. [CrossRef]

25. Jin, K.; Norris, K.; Vaziri, N.D. Dysregulation of hepatic fatty acid metabolism in chronic kidney disease. Nephrol. Dial. Transplant. 2013, 28, 313-320. [CrossRef] [PubMed]

26. Levey, A.S.; Stevens, L.A.; Schmid, C.H.; Zhang, Y.L.; Castro, A.F.; Feldman, H.I.; Kusek, J.W.; Eggers, P.; Van Lente, F.; Greene, T.; et al. A new equation to estimate glomerular filtration rate. Ann. Intern. Med. 2009, 150, 604-612. [CrossRef]

27. Kowalkowska, J.; Slowinska, M.A.; Slowinski, D.; Dlugosz, A.; Niedzwiedzka, E.; Wadolowska, L. Comparison of a full food-frequency questionnaire with the three-day unweighted food records in young Polish adult women: implications for dietary assessment. Nutrients 2013, 5, 2747-2776. [CrossRef] [PubMed]

28. Folch, J.; Lees, M.; Sloane Stanley, G.H. A simple method for the isolation and purification of total lipides from animal tissues. J. Biol. Chem. 1957, 226, 497-509. [PubMed]

29. Sledzinski, T.; Mika, A.; Stepnowski, P.; Proczko-Markuszewska, M.; Kaska, L.; Stefaniak, T.; Swierczynski, J. Identification of Cyclopropaneoctanoic Acid 2-Hexyl in Human Adipose Tissue and Serum. Lipids 2013, 48, 839-848. [CrossRef]

30. Nguyen, P.; Leray, V.; Diez, M.; Serisier, S.; Le Bloc'h, J.; Siliart, B.; Dumon, H. Liver lipid metabolism. J. Anim. Physiol. Anim. Nutr. (Berl.) 2008, 92, 272-283. [CrossRef]

31. Guillouzo, A.; Corlu, A.; Aninat, C.; Glaise, D.; Morel, F.; Guguen-Guillouzo, C. The human hepatoma HepaRG cells: A highly differentiated model for studies of liver metabolism and toxicity of xenobiotics. Chem. Biol. Interact. 2007, 168, 66-73. [CrossRef] [PubMed]

(C) 2019 by the authors. Licensee MDPI, Basel, Switzerland. This article is an open access article distributed under the terms and conditions of the Creative Commons Attribution (CC BY) license (http://creativecommons.org/licenses/by/4.0/). 